\title{
A simulation package for the signal pathway in absorbing media under linear perturbation
}

\author{
M.G.S. Ali and A. Raouf Mohamed \\ Physics Department, Faculty of Science, Minia University, \\ Egypt
}

(Received 12 November 1993)

\begin{abstract}
This paper describes a simple simulation of the signal pathway in an ultrasonic A-scan. It includes transient diffraction acoustic field, transducer response, and frequency dependent absorption in the field medium. The approach selected here aim to develop digital computer simulations of the physical processes that underlie these effects. The soft tissue is considered as a viscoelastic medium and the relaxation theory has been used to calculate the absorption data and dispersion in the tissue. The simulation is used to estimate the degradation in range of resolution due to wave absorption.
\end{abstract}

Keywords: Simulation, Absorption, Transducer, Resolution, Convolution

PACS number: 43.40 . Le

\section{INTRODUCTION}

The displayed images from ultrasonic scanning systems depend strongly on the characteristics of the overall signal path. These include the reflection or scattering processes that form the echoes, the characteristics of the transducers, the electronic systems, radiation coupling phenomena, and attenuation and phase velocity dispersion in the test medium. The ability to interpret complex images, or even to improve them by computational means, depends much on our understanding of the physics of wave propagation in the test medium, and on the transducer responses. It is possible to predict quantitatively the beam degradation observed in attenuating media, and to show that the strong influence of bandwidth on the frequency shift in attenuating media require careful design to arrive at an adequate compromise between pulse length (range resolution) and average frequency at different depths in the field (lateral resolution).

In this paper we present a simulation package of the signal pathway in a simple pulse-echo system operating into an absorbing medium under linear perturbation. It has enabled us to study the effects of attenuation on pulse waveforms, and the tradeoff between sensitivity and range resolution, taking into account electronic noise generated by the system. In our simulation, we have considered an unfocused single element pulse-echo transducer aligned with its face parallel to a flat lossless target in an absorbing medium. We assume simple impulse excitation to the transducer, and consider the received echo waveform and its frequency spectrum, at the terminals of the same transducer. The simulation of the signal path includes

(i) the non-uniform nature of the acoustic field, as expressed by the radiation coupling response from the transducer to the target and back again;

(ii) absorption and velocity dispersion, taking typical characteristics of soft biological tissues as an example;

(iii) the electro-acoustic properties of the transducer.

The approach taken is to assume that these three processes are linear, time invariant and separable from each other. The overall signal pathway is assumed to be the convolution of three impulse responses representing, respectively, the acoustic field, 
absorption and dispersion, and the transducer. Equivalently, the signal pathway can be considered in the frequency domain, as a multiplication of the transfer function representing each of the three phenomena. ${ }^{1)}$ In our simulation it is possible to take either a time domain or a frequency domain approach for each phenomenon, and to transfer to the other domain by means of fast Fourier transform (FFT).

\section{TRANSIENT DIFFRACTION IN THE ACOUSTIC FIELD}

Transient acoustic radiation from circular transducers is most simply formulated in terms of the planewave-edgewave hypothesis. ${ }^{2,3)}$ Starting with the Rayleigh integral, pressure at a point in the acoustic field is shown to consist of two componentsa plane-wave due to axial piston-line movement of the whole active face of the device; this is known as the face wave. It is followed by a diffracted wave emanating from a line source around the periphery of the piston. The two components are of opposite sign and of equal integral in the time domain, yielding zero response at zero frequency. For a transmission system with two circular transducers aligned coaxially with their faces parallel, and assuming no energy absorption in the medium between the two devices, the face wave applies a plane disturbance equally at all points on the receiver. The effect of the edge wave is to sweep across the receiver trans. ducer face, the device response being in proportion to the surface integral of instantaneous pressure on its face. For disc transducers of equal diameter the force coupling impulse response has been formulated by Rhyne4) as

$$
r(t)= \begin{cases}0 & 0 \leq t \leq t_{1} \\ \delta\left(t-t_{1}\right)-\frac{c^{2} t}{a^{2} \pi}\left[\frac{t_{2}{ }^{2}-t^{2}}{t^{2}-t_{1}{ }^{2}}\right]^{1 / 2} & t_{1} \leq t \leq t_{2} \\ 0 & t \geq t_{2}\end{cases}
$$

where $c$ is the wave propagation velocity, $a$ is the transducer radius, $t_{1}$ is the time of arrival of the face wave, and $t_{2}$ marks the termination of the edge wave contribution to the force coupling impulse response; $t_{2}$ corresponds to the furthest distance between points on the periphery of the transmitter and points on the periphery of the receiver.

$$
\begin{aligned}
& t_{1}=\frac{L}{c} \\
& t_{2}=\left(L^{2}+4 a^{2}\right)^{1 / 2} / c
\end{aligned}
$$

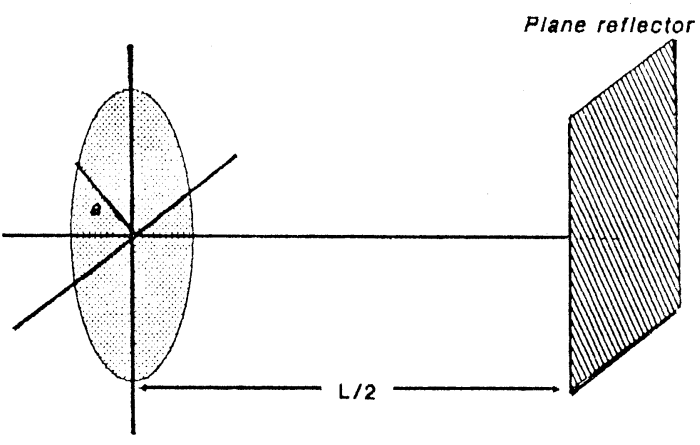

Fig. 1 A transmitting and receiving disk of radius a in an infinite baffle radiating to an infinite plane.

In the interval $\left(t_{1} \leq t \leq t_{2}\right)$, the first term $\delta\left(t-t_{2}\right)$ represents the face wave component, and the second term represents the edge wave. According to Rhyne, the formulation of Eq. (1) can be used to represent radiation coupling between a disc transducer to a plane reflector and back to the same disc transducer. Here, the round trip distance is $L$ and the transducer-target range is $L / 2$. The self-coupling of a disk radiating to and receiving from a planar target is defined by the geometry of Fig. 1. This method has been used to simulate the pulse-echo radiation coupling response in a system with a transducer of $10 \mathrm{~mm}$ diameter for a number of different target ranges.

\section{MEDIUM}

An ultrasound pulse is attenuated and phase is shifted when travelling through a medium. The attenuation can be described by the attenuation coefficient, which is usually strongly dependent on frequency. The attenuation of pressure waves and the resulting shift in wave velocity are important features in commonly used transmission media such as biological tissue. It is generally believed that the soft tissue can be treated as a viscous liquid for propagation of ultrasound. In this paper, soft tissue is considered as a viscoelastic liquid in which the elastic and viscous components are assumed to be in parallel. It has been shown ${ }^{5)}$ that, for a single relaxation frequency, the ultrasonic speed in the tissue is:

$$
c^{2}=\frac{K^{\prime}+(4 / 3) G^{\prime}}{\rho}
$$

and the absorption coefficient $\alpha$ is: 


$$
\frac{\alpha}{f^{2}}=2 \pi^{2}\left(\rho c^{3} \omega\right)^{-1}\left(K^{\prime \prime}+\frac{4}{3} G^{\prime \prime}\right)
$$

where $\rho$ is the mass density of the tissue and $\omega$ the angular frequency. The adiabatic modulus $(K)$ and shear modulus $(G)$ are complex and are frequency dependent:

$$
\begin{gathered}
K(\omega)=K^{\prime}+i K^{\prime \prime}=K_{0}+\frac{i K_{2} \omega \tau_{\mathrm{v}}}{1+i \omega \tau_{\nabla}} \\
G(\omega)=G^{\prime}+i G^{\prime \prime}=\frac{G_{\infty} i \omega \tau_{\mathrm{s}}}{1+i \omega \tau_{\mathrm{s}}}
\end{gathered}
$$

where $K_{0}$ is the value of bulk modulus as the frequency $\omega \rightarrow 0$, the relaxation modulus $K_{2}=K_{\infty}-$ $K_{0}, K_{\infty}$ is the value of the bulk modulus as $\omega \rightarrow \infty$, $\tau_{\mathrm{v}}$ the relaxation time for structural rearrangement in the medium, $\tau_{\mathrm{s}}$ is the shear relaxation time and $G_{\infty}$ is the high frequency shear modulus.

Acoustic attenuation due to shear relaxation invariably can not be accounted for by a single relaxation times instead a continuous distribution of relaxation times must be used. This is because the relaxation process arises from the finite time taken for molecules to diffuse between adjacent shearing layers in the medium. ${ }^{6)}$ The time taken for a particular molecule to diffuse depends upon its local environment which because of the random nature of medium is different for each molecule. Consequently the bulk behavior of the medium can only be described by a distribution of relaxation times and the real and imaginary parts of bulk modulus takes the form,

$$
K^{\prime}=K_{0}+K_{2} \int_{0}^{\infty} g\left(\tau / \tau_{\mathrm{v}}\right) \frac{\omega^{2}{\tau_{\vee}}^{2}}{1+\omega^{2}{\tau_{\mathrm{v}}}^{2}} d\left(\tau / \tau_{\mathrm{v}}\right)
$$

and

$$
K^{\prime \prime}=K_{2} \int_{0}^{\infty} g\left(\tau / \tau_{\mathrm{v}}\right) \frac{\omega \tau_{\mathrm{v}}}{1+\omega^{2}{\tau_{\mathrm{v}}}^{2}} d\left(\tau / \tau_{\mathrm{v}}\right)
$$

where $g\left(\tau / \tau_{\mathrm{v}}\right)$ represents the distribution of relaxation times and is normalised such as

$$
\int_{0}^{\infty} g\left(\tau / \tau_{\mathrm{v}}\right) d\left(\tau / \tau_{\mathrm{v}}\right)=1
$$

The real and imaginary part of the shear modulus can be written in a like manner when a distribution of relaxation times exists. The simulation relationships of Eqs. (2) and (3) can be used to calculate the dispersion and absorption coefficient for tissue by Gaussian and Cole Davidson distribution function. ${ }^{7)}$

The influence of attenuation and dispersion in the transmission medium is evaluated by determining the complex wave number $K$,

$$
K(\omega)=\omega / c(\omega)-i \alpha(\omega)
$$

The transfer of the absorption process can be written as

$$
T(\omega, L)=e^{-i K(\omega) L}
$$

where $L$ is the distance from transmitter to target and back again.

\section{THE TRANSDUCER RESPONSES}

The transducer strongly affects the performance of an ultrasound imaging system. Because there is a strong relationship between the properties of the transducer and the quality of the resulting image, a variety of techniques has been developed for modelling transducer behaviour, and hence predicting its contribution to the overall system response. There are many approaches to the modelling of ultrasonic piezoelectric transducers. ${ }^{8-12)}$ Hargreaves $^{11)}$ has proposed a more complete model for predicting the behaviour of a multilayered piezoelectric transducer connected to any arbitrary electrical load. It uses $z$-transform techniques ${ }^{12)}$ to provide a discrete time implementation of the original frequency domain equivalent circuit of Mason (see Berlincourt et al. ${ }^{13)}$ ). His computer model is split into two parts. First, the basic transfer function of the loaded piezoelectric element is evaluated in discrete form using the $z$-transform technique. Secondly, the discrete time responses so obtained are then processes by a nested set of digital filter operations that simulate reverberation in the coupling layers connected to the device active element, and backing and load media connected to these. ${ }^{1)}$

The above simulation was applied using typical data for transducer constructed with a load zirconate titanate (PZT-5A) piezoelectric element, ${ }^{13)}$ with tungsten-epoxy $\left.{ }^{14}\right)\left(z=35 \times 10^{6} \mathrm{~kg} / \mathrm{m}^{2}\right.$-s $)$ back block, and a quarter-wave matching layer of epoxy. The transmitter response is obtained for a $10 \mathrm{~mm}$ diameter pulse-echo transducer of $5 \mathrm{MHz}$ centre frequency. The impedance of the generator is assumed to be $5 \mathrm{Ohms}$ resistance. Figure 2 shows the expected waveform at the transducer terminals when the device acts as a receiver; that is, in pulseecho mode with no radiation coupling, target or other field effects. Figure 3 shows the pulse-echo transducer frequency response of Fig. 2. From this figure, it can be seen that the pulse-echo trans- 


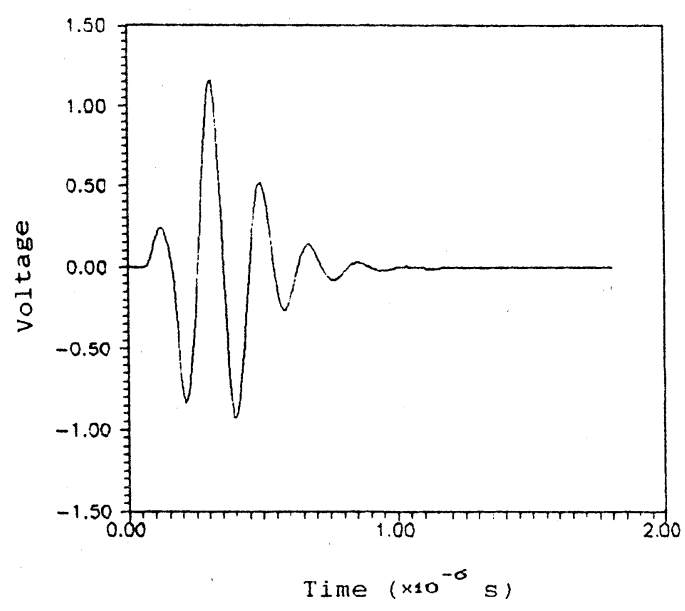

Fig. 2 The voltage at the transducer terminal when an echo is received from a plane reflector in a lossless medium without consideration of radiation coupling.

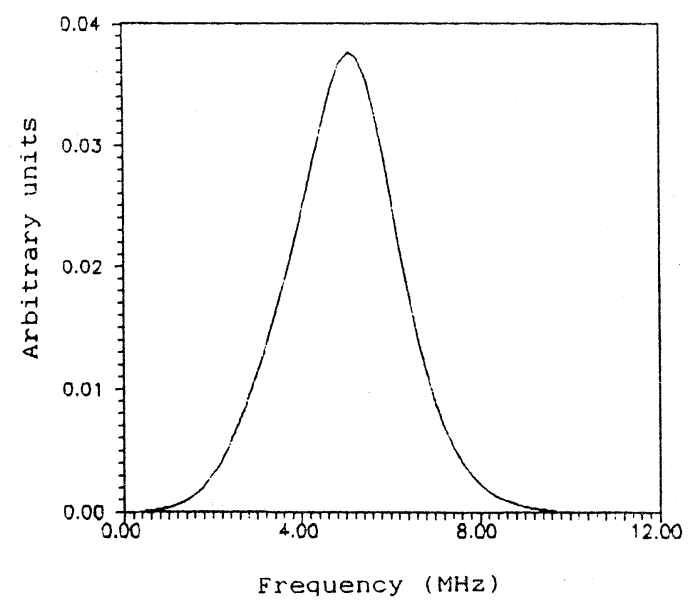

Fig. 3 Frequency spectrum of pulse echo response of Fig. 2.

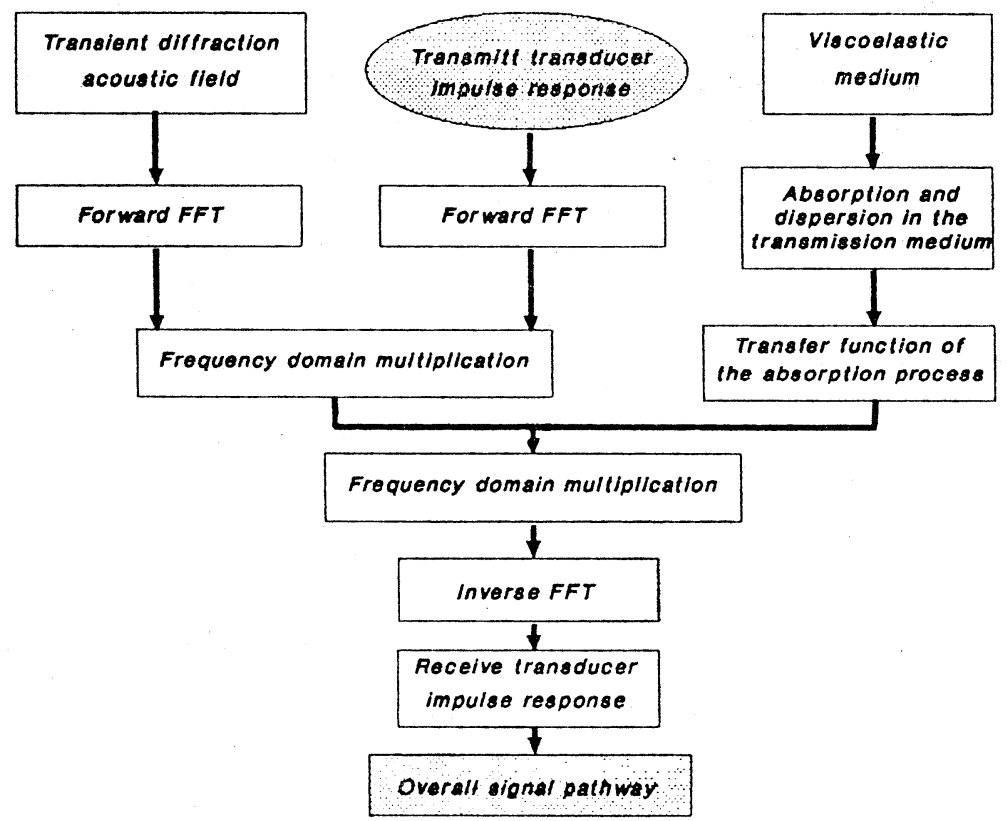

Fig. 4 Function blook diagram of the overall system model.

ducer yield $6 \mathrm{~dB}$ bandwidth of $53 \%$ of the centre frequency.

\section{OVERALL SYSTEM RESPONSE}

The signal pathway of a pulse-echo system consists of the responses of the transducer in transmis- sion and reception combined with the radiation coupling response, and the impulse response due to absorption and dispersion in the test medium. A function block diagram of the overall system model is described in Fig. 4. The signal transfer process can be modelled by a series of convolution. If 
$x(t)$ is the input voltage drive to the pulse-echo transducer and $y(t)$ is the output voltage at the transducer terminals in reception, then

$$
y(t)=f_{\mathrm{T}}(t) * r(t) * g(t) * f_{\mathrm{R}}(t) * x(t)
$$

where $*$ represent the convolution integral, given by

$$
p(t) * q(t)=\int_{-\infty}^{-\infty} q(\tau) p(t-\tau) d \tau
$$

The symbols have the following meanings:

$$
\begin{aligned}
f_{\mathrm{T}}(t)= & \text { transmission transducer impulse response; } \\
r(t)= & \text { impulse response of transient radiation } \\
& \text { coupling; } \\
g(t)= & \text { impulse response of absorption and dis- } \\
& \text { persion in the test medium; } \\
f_{\mathrm{R}}(t)= & \text { receiver transducer impulse response. }
\end{aligned}
$$

The real frequency model is obtained from the impulse responses by Fourier transformation and replacing the time domain convolution by frequency domain multiplication, thus

$$
Y(j \omega)=F_{\mathrm{T}}(j \omega) R(j \omega) G(j \omega) F_{\mathrm{R}}(j \omega) X(j \omega)
$$

where

$$
Y(j \omega)=\int_{-\infty}^{+\infty} y(t) e^{-j \omega t} d t
$$

or equivalently

$$
\begin{array}{lll}
Y(j \omega) \leftrightarrow y(t) & F_{\mathrm{T}}(j \omega) \leftrightarrow f_{\mathrm{T}}(t) & G(j \omega) \leftrightarrow g(t) \\
R(j \omega) \leftrightarrow r(t) & F_{\mathrm{R}}(j \omega) \leftrightarrow f_{\mathrm{R}}(t) & X(j \omega) \leftrightarrow x(t)
\end{array}
$$

\section{SIMULATION RESULTS}

The simulations were carried out by multiplying the transfer functions of the two transducer responses by the radiation coupling response and the transfer function of the transmission medium in the frequency domain. The resulting response was then converted back into the discrete time domain by inverse Fourier transformation. In order to investigate the combined effects of the transducer and the phenomena described above on scanning system performance the simulation was applied to a medium of soft biological tissue (see Ref. 15)). The pulse-echo response of the transducer shown in Fig. 2 has been combined with the radiation coupling and medium absorption responses for a depth of $10 \mathrm{~cm}$ in biological tissue. The overall time domain response at the receiver terminal is shown in Fig. 5. From this figure it can be seen that the duration of the signal increases after propagation in the loss medium. Figure 6 shows the spectrum of

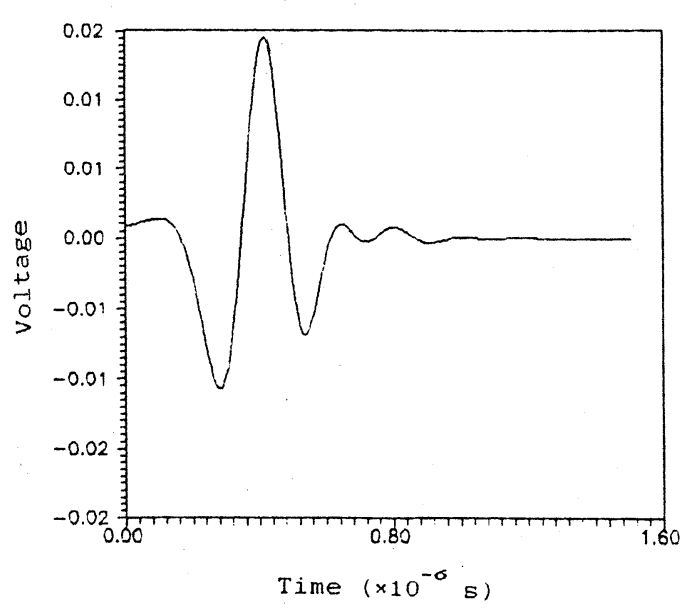

Fig. 5 Time domain pulse-echo response of $5 \mathrm{MHz}$ transducer operating into soft tissue with an ideal flat target at a range of $5 \mathrm{~cm}$. This response includes the effects of the transducer, absorption, velocity dispersion, and radiation coupling.

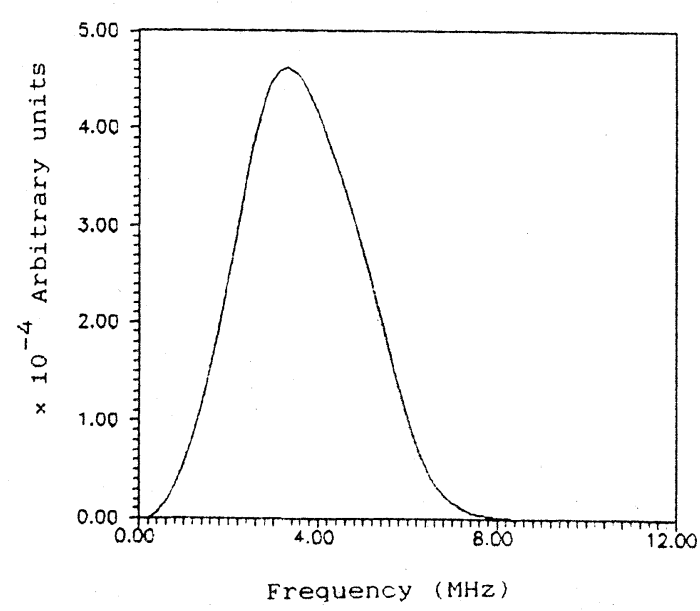

Fig. 6 Frequency spectrum of pulse-echo response from an ideal plane target in soft tissue at $5 \mathrm{~cm}$ range.

the original pulse after travelling $10 \mathrm{~cm}$ through biological tissue (pulse-echo range $5 \mathrm{~cm}$ ). Figure 7 shows the shift of the pulse centre frequency. It is clear from this figure that the receiving transducer can not work in resonance because the frequency spectrum of the receiving pulse depends on the filtering effects of absorption and depth. This causes sensitivity loss and, at the same time the 


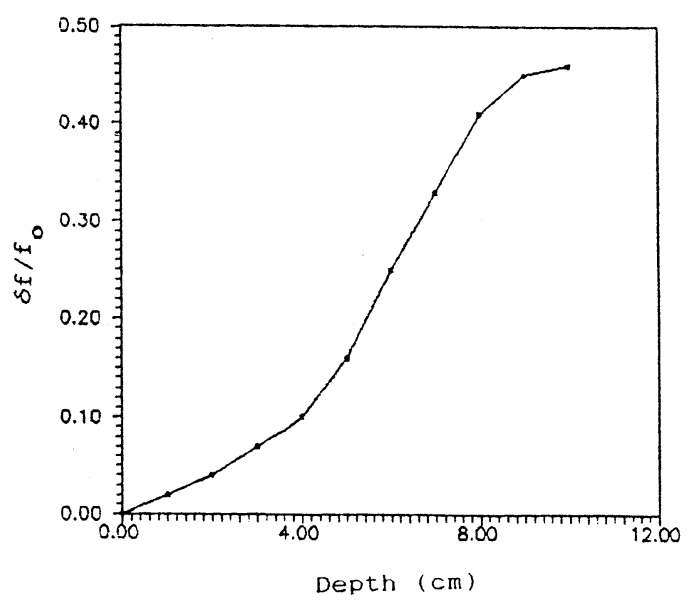

Fig. 7 Change in received pulse centre frequency relative to that obtained in a lossless medium for an echo signal from an ideal plane target in soft tissue at ranges between 0 and $10 \mathrm{~cm}$.

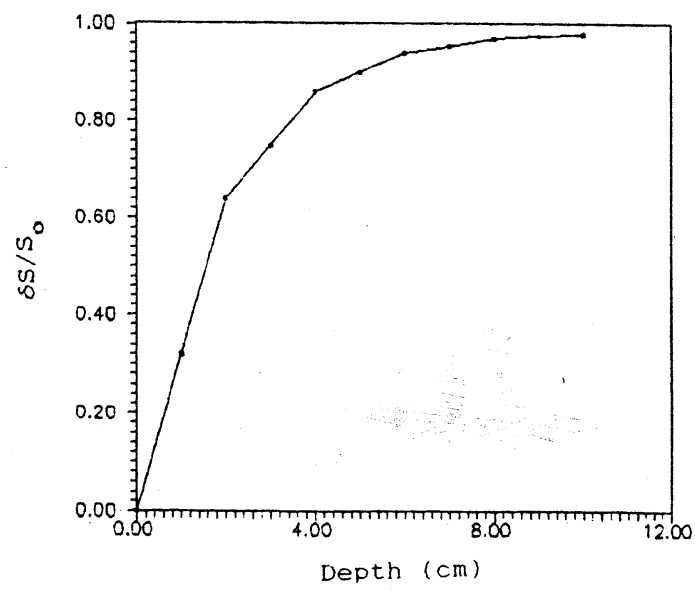

Fig. 8 Loss of sensitivity in terms of relative change in pulse height versus reflector range for the system of Fig. 7.

resolution is lowered by the dominance of longer wavelengths in the acoustic signals.

The sensitivity loss is defined as the decrease in the maximum height of the acoustic pulse at the receiver. If $S_{0}$ represents the maximum height of the original pulse transmitted by the transducer and $\delta S$ represents the decrease in pulse height alter travelling through biological tissue, then $\delta S / S_{0}$ represents the loss of sensitivity. Figure 8 shows the loss of

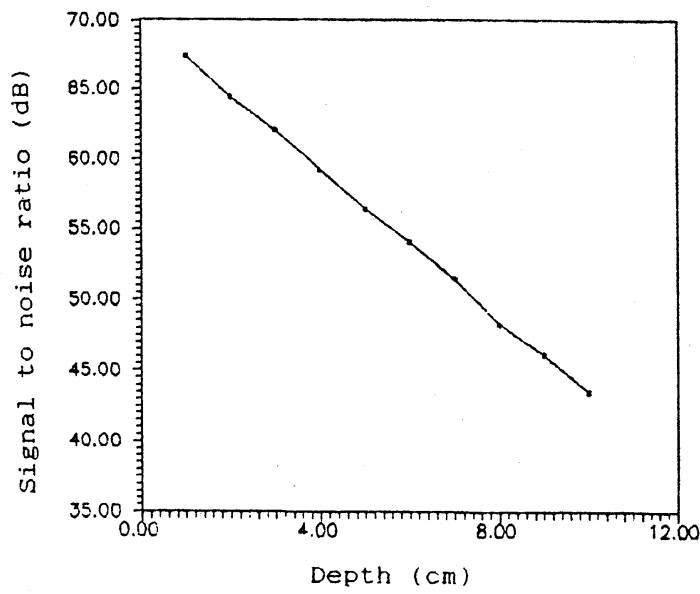

Fig. 9 Signal to noise ratio (SNR) at different depth in frequency domain.

sensitivity at different depths in soft tissue. Figure 9 shows the calculation of signal to noise ratio (SNR) for soft tissue at different depth in frequency domain. It is clear from the above calculation that the noise increases as the depth increases. Our results show that the sensitivity reduces and the lateral resolution is lost as the depth increases in attenuating media. Finally, the transducer and absorption responses dominate the overall signal pathway response, while radiation coupling and velocity dispersion, although interesting from a scientific viewpoint, could be omitted from the model with little change in the principle conclusions.

\section{CONCLUSIONS}

This paper has described a simple simulation of the signal pathway in a pulse-echo A-scan system. The model includes the transient radiation coupling process, frequency dependent absorption in the test medium, and piezoelectric transducer insertion effects. The soft tissue is considered as a viscoelastic liquid or a voigt body in which the elastic and viscous components are assumed to be in parallel. The simulation has been used to estimate the degradation in longitudinal resolution that results from frequency dependent signal loss. For a given medium and imaging depth it provides a means by which the transducer properties can be defined quantitatively to satisfy the range resolution requirements of a scanning system. The calculation of the signal to noise ratio and its trade-off with 
resolution has been developed.

\section{REFERENCES}

1) M. G. S. Ali and A. R. Mohamed, "A simulation of pulse-echo amplitude scan signal formation in absorbing media," Ultrasonics 30, 311 (1992).

2) O. G. Kozina and G. I. Makarov, "Transient processes in the acoustic fields of special piston membrances," Sov. Phys. Acoust. 8, 49 (1962).

3) P. R. Stepanishen, "Transient radiation from piston in an infinite planar baffle," J. Acoust. Soc. Am. 49, 1629 (1971).

4) T. L. Rhyne, "Radiation coupling of a disk to a plane and back, or a disk to a disk," J. Acoust. Soc. Am. 61, 318 (1977).

5) A. J. Matheson, Molecular Acoustics (Wiley-Interscience, London, 1971).

6) H. Pauly and H. P. Schwan, "Mechanism of absorption of ultrasound in liver Tissue," J. Acoust. Soc. Am. 50, 692 (1971).

7) R. Meister, C. J. Marhoeffer, R. Sclamand, L. Cotter and T. Litovitz, "Ultrasonicviscoelastic properties of associated liquids," J. Appl. Phys. 31, 854 (1960).

8) E. K. Sitting, "Transmission parameters of thickness driven piezoelectric transducers arranged in multilayer configurations," IEEE Trans. Sonics Ultrason. SU-14, 167 (1967).

9) G. K. Leis, "A matrix technique for analyzing the performance of multilayered front matched and backed piezoelectric ceramic transducers," Acoust. Imaging 8, 395 (1980).

10) Hayward, C. J. Macleod and T.S. Durrani, "A systems model of the thickness mode piezoelectric transducer," J. Acoust. Soc. Am. 76, 367 (1984).

11) M. L. Hargreaves, Ph. D Thesis, 1988, Keele Univ., Staffs, ST5 5BG, UK (1988).

12) R. E. Challis and J. A. Harrison, "Rapid solutions to the transient response of piezoelectric elements by $z$-transform techniques," J. Acoust. Soc. Am. 74, 1673 (1983).

13) D. A. Berlincourt, D. R. Curran and H. Jaffe, "Piezoelectric and Piezomagnetic materials and their function in transducers," in Physical Acoustics 1A, W. P. Mason, Ed. (Academic, New York, 1964).

14) J. W. Hunt, M. Akditi, and F. S. Foster, "Ultrasound transducers for pulse-echo medical imaging," IEEE Trans. Biomed. Eng. BME. 30, 454 (1983).

15) E. L. Madsen, H. J. Sathoff, and J. A. Zagzebski, "Ultrasonic shear wave properties of soft tissues and tissuelike materials," J. Acoust. Soc. Am. 74, 1346 (1983). 